\title{
¿EL PROCESO DE ENFERMERÍA DE AUTOCUIDADO GENERA MAYOR ADHERENCIA AL TRATAMIENTO EN TRASPLANTE RENAL?*
}

Fanny Moreno Rubio**, Esmelly Vargas Montaña**

\section{Resumen}

El proceso de enfermería es un instrumento creado por los profesionales de esta disciplina para mejorar la calidad de atención y favorecer los resultados de los pacientes. Cuando se utilizan los diagnósticos de enfermería para establecer objetivos y seleccionar las intervenciones, el resultado es un plan de cuidados holístico e individualizado que responderá a las necesidades específicas del paciente. Es necesario mostrar las evidencias de las acciones a seguir con el enfermo trasplantado observando el entorno, medio sociocultural y recursos físicos y humanos, orientados desde la experticia y el conocimiento científico. La presente revisión pretende iidentificar y evaluar de manera sistemática la literatura referente a la adherencia al tratamiento de aquellos con trasplante renal, a partir de procesos de cuidado en enfermería fundamentados en el modelo de autocuidado.

Palabras clave: proceso de enfermería, autocuidado, adherencia, trasplantes.

Abreviaturas: AC, autocuidado.

\section{DOES THE SELF-CARE NURSING PROCESS ENHANCE ADHERENCE TO TREATAMENT IN RENAL TRANSPLANT PATIENTS?}

\section{Abstract}

The nursing process is a tool developed by nursing professionals to improve quality of care and patient outcomes. Nursing diagnoses are used to determine objectives and for intervention selection thus rendering an holistic and personalized plan which will satisfy the specific needs of each patient. Evidences of the regimen to be followed with patients who have undergone transplants must be identified considering aspects such as, setting, sociocultural environment and physical and human resources, oriented by scientific expertise and knowledge. This article pretends to identify and evaluate literature on patients who have undergone renal transplants and their adherence to treatment, conducting a systematic review based on the self-care model nursing process.

Key words: nursing process, self-care, adherence, transplants.

Fecha recibido: octubre 26 de 2009 - Fecha aceptado: enero 4 de 2010

* Trabajo presentado en Congreso Nacional de Enfermeros de Trasplante, julio de 2009, Cali, Colombia.

\footnotetext{
** Coordinadora Unidad de Trasplantes, Hospital de San José, Bogotú DC.

*** Enfermera auditora Oficina de Calidad, Hospital de San José, Bogotá DC.
} 
Escenario clínico. El trasplante renal representa el tratamiento de elección para los pacientes con insuficiencia renal crónica y sus excelentes resultados se ven reflejados en la función inmediata del injerto, la mejor calidad de vida y que en corto tiempo pueden desaparecer los problemas adquiridos durante la enfermedad renal crónica, como anemia o lesiones en huesos, músculos y nervios. Para un pequeño porcentaje el proceso de recuperación en el posoperatorio es más lento, lo que podría ocasionar la aparición de complicaciones como infecciones, rechazo del injerto o reaparición de la enfermedad de base, y podría presentarse una variación del pronóstico. El enfermero responsable del programa de trasplantes debe entrevistar al enfermo y su familia para identificar las necesidades informativas y diligenciar el instrumento de valoración clínica.

Con base en la información recolectada y las necesidades identificadas el enfermero planea y ejecuta el cuidado de los pacientes en las fases del trasplante renal: en el pretrasplante se explica de forma personalizada el proceso de evaluación e ingreso a la lista de espera y se define el tipo de donante; en el postrasplante se orienta el cuidado del paciente, se brinda educación sobre el manejo de medicamentos, hábitos, alimentación y asistencia a los controles. Es importante que el profesional sustente cuidados generales con validez científica que demuestren la importancia de las acciones a seguir, aplicando el proceso de enfermería fundamentado en el AC.

En la Unidad de Trasplantes del Hospital de San José se realizaron durante el 200831 trasplantes y el equipo de enfermería al observar problemas en la adherencia al tratamiento, decidió implementar en veinte pacientes trasplantados un programa fundamentado en el modelo de AC, basado en la teoría de D. Orem el cual incluye: valoración, diagnóstico e intervenciones de enfermería para el cuidado posoperatorio en un lapso de seis meses. Antes de iniciar la experiencia surgió el siguiente interrogante: ¿El proceso de enfermería fundamentado en el modelo de AC genera mayor adherencia al tratamiento en pacientes con trasplante renal?

\section{Introducción}

El propósito de la presente revisión crítica es identificar y evaluar de manera sistemática la literatura referente a la adherencia al tratamiento en pacientes trasplantados a partir de procesos de enfermería fundamentados en el modelo de AC, entendido como el conjunto de acciones que realiza la persona para controlar los factores internos y externos que puedan comprometer su vida y desarrollo. Según Orem, el AC no es innato, es una conducta aprendida a través de las relaciones interpersonales, la comunicación con la familia, la escuela y los amigos, que se da a lo largo de la vida, en el que el agente de AC es la persona que lleva a cabo dichas acciones. El modelo hace hincapié en que la función del enfermero surge sólo cuando el paciente es incapaz de satisfacer por sí mismo sus necesidades de AC. La intervención suele estar dirigida a conservar la salud, prevenir la enfermedad o restablecer la salud, y puede incluir actividades realizadas para el paciente o en colaboración con él. En este sentido el proceso de cuidado de enfermería basado en la teoría de AC debe contemplar una valoración, un diagnóstico, una intervención de enfermería para realizar y fijar los resultados esperados.

Estrategia de búsqueda: la revisión de la literatura para identificar y resumir la evidencia referente a los cambios en la adherencia al tratamiento de pacientes trasplantados a partir de procesos de cuidado en enfermería, fundamentados en el modelo de AC fue realizada en Crochane. Se empleó una estrategia que incluyó términos MESH y palabras clave para identificar revisiones sistemáticas, ensayos clínicos aleatorios, estudios controlados, series de tiempo, estudios de antes y después, observacionales, y estudios cualitativos y descriptivos. Además, se verificaron las listas de referencias de las publicaciones encontradas para identificar adicionales. Se incluyeron artículos en idiomas originales (inglés y español).

Análisis: ocho referencias se identificaron en la búsqueda. No se encontraron estudios aleatorios, controlados, de antes y después o series de tiempo que hicieran referencia a estrategias de cuidado en enfermería basadas en la teoría del AC para mejorar la adherencia al tratamiento en los pacientes trasplantados. Se hallaron dos estudios descriptivos prospectivos. Uno de ellos realizado en $2005^{1}$ que intentó determinar los conocimientos adquiridos por el paciente trasplantado renal hasta el día del alta hospitalaria, en cuanto a la toma de los medicamentos y el AC. Su análisis se basó en el modelo de AC de Dorotea Orem. Se partió de las recomendaciones 
contenidas en la Guía del paciente con trasplante renal e incluyó a los receptores de un primer rasplante; se excluyeron los trasplantados con donante vivo y los retrasplantes. A los participantes se les aplicó un cuestionario de veinte preguntas cerradas distribuidas en tres bloques, el primero valoró la toma correcta de los medicamentos, el segundo los cuidados referentes a higiene, ejercicio y cuidados específicos del trasplante y el tercero interrogó dónde y cuándo se les entregó la cartilla con la información acerca de sus cuidados postrasplante. En este trabajo se concluye que hay un déficit importante de conocimientos sobre $\mathrm{AC}$ al salir del hospital ras recibir un trasplante renal, poniendo en peligro la supervivencia del injerto. Así mismo se identificaron falencias en la guía elaborada, concluyendo que la formación educativa del paciente no influye para asimilar las recomendaciones contenidas en la publicación razón por la cual sugieren que todos deben recibir educación adicional para el cuidado del rasplante.

El orro estudio descriptivo y prospectivo fue realizadoen la unidad de hospitalización de nefrología del Hospital Universitario de Canarias. ${ }^{2}$ Se tomaron 140 pacientes de un total de 452 rasplantados que cumplían con criterios de inclusión (recibieron injerto del 27/10/03 al 30/01/ 06) y elaboraron un listado de diagnósticos de enfermería idóneos basados en NANDA y prevalentes en su unidad, ante lo cual concluyeron que el uso de planes de cuidados es una práctica reciente que permite eliminar riesgos y prestar una atención global sin correr el riesgo de obviar cuidados e intervenciones que serían beneficiosas. Aunque la generación de "guías para el paciente trasplantado" son útiles, se debe tener en cuenta que cada individuo es único y por tanto hay que adecuar el plan de cuidados.

Se revisó otro estudio de adhesión terapéutica realizado en 1993 en dos fases. ${ }^{3}$ En la primera se hizo una revisión rerospectiva de la historia clínica de 394 rasplantados, con un riñón funcionante al menos durante tres meses. En la segunda fase se realizó una encuesta de forma anónima entre los receptores de un injertorenal que evaluó la adhesión al ratamiento. Se definieron res grupos: uno, la falta de adhesión al ratamiento inmunosupresor, dos, la adhesión incompleta cuando se presentaba descontrol en los horarios de toma de medicamentos y tres, no seguimiento de la dieta instaurada. La encuesta fue realizada por el profesional de enfermería al ingreso del paciente y antes del egreso hospitalario tras el trasplante, y se evaluó si los pacientes realizaban correctamente la autodosificación de la ciclosporina. Dentro de las principales conclusiones está la dificultad para cuantificar el grado de incumplimiento de los enfermos al no existir un método directo de certeza. Así mismo, menciona una correlación positiva entre el abandono del ratamiento y la pérdida del injerto.

En general los estudios revisados reconocen la importancia de la implementación de estrategias de cuidado en enfermería para mejorar la adherencia a los tratamientos en los pacientes trasplantados, aunque sólouno de ellos hace énfasis en las centradas en la teoría de AC propuesta por Dorotea Orem. Las principales estrategias reportadas son: valoración, diagnóstico, consejería, entrega de información escrita y realización de planes educativos. Dentro de éstas las más utilizadas para mejorar la adhesión al tratamiento brindar información escrita y tratar de adecuar el tratamiento a los hábitos del paciente. Al revisar la literatura se anotan cambios en la adherencia al tratamiento como respuesta al plan de atención de enfermería que incluya educación al paciente ${ }^{4}$ y aunque cada una de las estrategias mencionadas reportan potencialidades, siguen siendo limitadas ante el problema de la no adherencia a los tratamientos.

Por su parte, las estrategias implementadas en el Hospital de San José basadas en la teoría de $\mathrm{AC}^{5}$ incluyen una valoración inicial de enfermería al paciente aplicando un instrumento con base en los patrones funcionales, determinando diagnósticos de enfermería y realizando la intervención requerida de acuerdo con las necesidades identificadas. En el postrasplante, el día de alta de la institución se entrega una orientación de los horarios de los medicamentos y una cartilla con los cuidados necesarios y las señales de alarma. Una semana después del alta hospitalaria se aplica una encuesta que evalúa la educación dada. El $85 \%$ de los pacientes responden de manera adecuada, pero el $15 \%$ requiere hasta dos reforzamientos más de la educación para lograr los resultados esperados en términos de adherencia al tratamiento. 


\section{Conclusiones}

Nuestra revisión crítica permite concluir que en la actualidad existe un especial reconocimiento de la importancia de implementar estrategias de cuidado en enfermería para mejorar la adherencia a los tratamientos de los pacientes trasplantados, pero se observa un vacío de conocimiento importante en el tema de la orientación de dichas estrategias y su aplicación. A partir de la experiencia exitosa de seguimiento al paciente realizado en el Hospital de San José, se podría sugerir que la aplicación del modelo de AC de Dorotea Orem genera mayor adherencia en el paciente trasplantado. Sin embargo, se requiere generar procesos de investigación que permitan reforzar el mencionado resultado.

\section{Referencias}

1. Escofet Gómez R, Salillas Adot E, et al. ¿Qué conocimientos ha adquirido el paciente rasplantado renal al alta hospitalaria?. Rev. SEDEN [serie en Intemet]. [citado 27 Feb. 2010];[aprox. 4 p.]. Disponible en: http://www. revistaseden.org/files/74.pdf

2. Martín Fleitas AE, Marrero Fernández MP. Los diagnósticos de enfermería en el trasplante renal, una base esencial para un buen plan de cuidados. Rev. SEDEN [serie en Internet]. [citado 27 Feb. 2010]; [aprox. 4 p.]. Disponible en: http://www.revistaseden.org/files/66.pdf

3. Orofino L, Marcén R, Quereda C, Burgos J, Ortuño J. Adhesión al tratamiento del paciente trasplantado renal. Nefrología [serie en Internet]. 1994 [citado 27 Feb. 2010]; 14(3): [aprox. 4 p.]. Disponible en: http://www. revistanefrologia.com/mostrarfile.asp?id=2366

4. Ferrero Hidalgo S. Cuidados de enfermería en el trasplante renalbasados en el modelo de Virginia Henderson $-1^{\circ}$ trimestre 1996. Rev. SEDEN [serie en Internet]. [citado 27 Feb. 2010]; [aprox. 4 p.]. Disponible en: http:// www.revistaseden.org/files/art524_l.pdf

5. Implementación de un modelo de cuidado de enfermería en el programa de trasplante renal del Hospital de San José. Fanny Moreno Rubio, $2^{\circ}$. Semestre 2008.

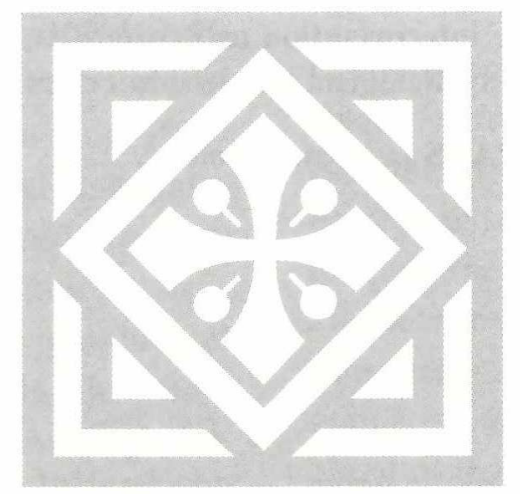

